\title{
Simulations of Laser Propagation and Ionization in l'OASIS Experiments
}

\author{
D. A. Dimitrov*, D. L. Bruhwiler*, W. Leemans ${ }^{\dagger}$, E. Esarey ${ }^{\dagger}$, P. Catravas ${ }^{\dagger}$, \\ C. Toth ${ }^{\dagger}$, B. Shadwick ${ }^{\dagger}$, J. R. Cary* and R. Giacone** \\ ${ }^{*}$ Tech-X Corporation \\ ${ }^{\dagger}$ Lawrence Berkeley National Laboratory \\ ${ }^{* *}$ University of Colorado
}

\begin{abstract}
We have conducted particle-in-cell simulations of laser pulse propagation through neutral He, including the effects of tunneling ionization, within the parameter regime of the l'OASIS experiments $[1,2]$ at the Lawrence Berkeley National Lab (LBNL). The simulations show the theoretically predicted [3] blue shifting of the laser frequency at the leading edge of the pulse. The observed blue shifting is in good agreement with the experimental data. These results indicate that such computations can be used to accurately simulate a number of important effects related to tunneling ionization for laser-plasma accelerator concepts, such as steepening due to ionization-induced pump depletion, which can seed and enhance instabilities. Our simulations show self-modulation occurring earlier when tunneling ionization is included then for a pre-ionized plasma.
\end{abstract}

\section{INTRODUCTION}

The quest to understand the fundamental nature of matter requires ever higher energy particle collisions, which in turn leads to ever larger and more expensive particle accelerators. Plasma-based accelerators can sustain electron plasma waves (EPW) with longitudinal electric fields on the order of the nonrelativistic wave breaking field, $E_{0}=c m_{e} \omega_{p} / e$, where $\omega_{p}=\sqrt{4 \pi n_{e} e^{2} / m_{e}}$ is the plasma frequency at an electron density $n_{e}$ (see Ref. [4] for a review of plasma-based accelerator concepts). For $n_{e}=10^{18}$ $\mathrm{cm}^{-3}$, the electric field is $E_{0} \cong 100 \mathrm{GV} / \mathrm{m}$ - several orders of magnitude higher than for conventional structures-providing hope for reaching new energy regimes.

In many cases, the laser pulse must create its own plasma via field-induced tunneling ionization of a neutral gas. Tunneling ionization due to laser pulse propagation in a neutral gas leads to frequency up-shifting (blue shifting) of the laser pulse, as has been observed and analyzed by several groups [5, 6]. A nonlinear expression for the frequency upshift was derived analytically by Esarey et al. [3]. Recently, Leemans et al. have proposed and used ionization-induced blue shifting as a method for determining optimized compression of the laser pulses in a chirped-pulse amplification laser system $[1,2]$.

The tunneling ionization has important ramifications to the intensively studied laser wakefield accelerator (LWFA) concept. In particular, the leading edge of the laser pulse can steepen due to ionization-induced pump depletion, which can seed and enhance the self-modulation instability [7]. Also, electron density gradients in the transverse wings 
of the pulse refract some energy away and modify the transverse profile via ionizationinduced refraction [8].

In this paper, we present results from computer simulations on laser propagation and tunneling ionization. These results are compared with data from l'OASIS experiments. The simulations provide valuable insight and diagnostics into the physics of laser frequency up-shifting due to ionization.

In the next section we briefly describe the particle-in-cell code we used. Our code implementation of tunneling ionization is then presented. The detailed parameters from both the experiments and the simulations are given and the results of the simulations are described. We compare tunneling ionization with preionized plasma simulations in the last section. This comparison shows the effects that are characteristic for the tunneling ionization, such as frequency up-shifting and pump depletion, are not present in the preionized plasma simulations. We conclude with a summary of the results and directions for future work.

\section{THE OOPIC PARTICLE-IN-CELL CODE}

The object-oriented particle-in-cell (OOPIC) $[9,10]$ code started as a pioneering effort to apply object oriented techniques to plasma simulation codes. OOPIC is a highperformance 2-D PIC code, with support for $x-y$ (slab) and $r$ - $z$ (cylindrical) geometries. It is written in $\mathrm{C}++$ and is ported to some of the most commonly used operating systems: Linux/Unix, and Windows. OOPIC runs on a number of different hardware platforms including x86 clusters (Linux based), IBM SP (POWER family of processors), and Cray T3E. The OOPIC Pro version of the code, distributed and supported by Tech$\mathrm{X}$ Corporation, provides on top of the computational physics kernel of the code, an intuitive graphical user interface based on the freely available QScimpl [11] scientific graphics package. OOPIC Pro is the only version of the code that runs on Windows with a graphical user interface. For advanced use, the OOPIC code can be steered completely automatically by running it without the graphical user interface in a batch mode using scripts. This is particularly helpful for running multi-process production jobs on supercomputers.

The message passing interface (MPI) is used in OOPIC to take advantage of massively parallel, symmetric multiprocessor and distributed architectures. The code has demonstrated good linear scaling up to 64 processors on IBM SP. It is routinely used with 16 and 32 processors on the Cray T3E, and with 8 processors on the Tech-X Corporation Linux/Athlon cluster. We are currently developing a separate library with the ionization algorithms from OOPIC. This will enable us to do 3-D PIC/Fluid ionization simulations using the VORPAL code $[12,13]$. VORPAL has extensive capabilities for PIC/Fluid simulations in multiple dimensions, including 3-D, and runs efficiently in a multi-processing mode.

OOPIC also includes volumetric and surface plasma injection, including thermionic and field emission models. Particle statistics can be collected at arbitrary surfaces. A Monte Carlo collision (MCC) technique [14] allows multiple background gases at arbitrary partial pressures. The features described are all adjustable from the input file 
using MKS or arbitrary units for input parameters. OOPIC has capabilities to simulate impact and tunneling ionization processes for a number of gases such as $\mathrm{H}, \mathrm{He}, \mathrm{Li}$. In the next section, we describe briefly how we have implemented tunneling ionization in OOPIC.

\section{IMPLEMENTATION OF TUNNELING IONIZATION IN OOPIC}

The theory of tunneling ionization in a strong electromagnetic field was initially developed by Keldysh [15]. He derived formulas for the ionization probability of Hydrogenlike atoms of gases and for crystals. The theory was later extended to ionization from excited electron states [16], to complex atoms and ions, and for radiation with different polarizations [17]. Recent experimental results together with the physics of tunneling ionization are reviewed by Delone and Krainov [18].

The tunneling ionization probability rate is derived using the adiabatic and quasiclassical (WKB) approximations [19]. The conditions for the applicability of these approximations impose bounds from above on the laser frequency $\omega$ :

$$
\omega \ll E_{i},
$$

where $E_{i}$ is the energy of the tunneling electron in the atom, the peak electric field $E_{0}$ :

$$
E_{0} \ll E_{a},
$$

where $E_{a}$ is the atomic electric field, and their ratio. The condition on their ratio is related to the so called Keldysh parameter $\gamma \equiv \omega \sqrt{2 E_{i}} / E_{0}$ and is usually expressed through it:

$$
\gamma^{2} \ll 1
$$

All the parameters in these conditions are in atomic units.

The simulation of tunneling ionization processes in OOPIC first initializes the relevant parameters that determine the regime of applicability of the tunneling ionization theory. Then, at each time step the probability rates for such ionization processes are calculated, the necessary ions and electrons are created while the neutral gas densities and other particles that undergo ionization are updated in each grid cell. We have implemented the formulas for tunneling ionization probability rates and the more precise criteria for their applicability as given by Amosov, Delone, and Krainov [17, 18]. The ions and electrons created at each time step in a considered grid cell, due to tunneling ionization, are added to the simulation with zero momenta.

This algorithm does not strictly preserve energy, since the field loses energy to ionize a particle (atom or ion that can be further ionized) and to create a new ion and electron separated by a small distance. However, the field in our simulation does lose energy to separate the newly added ions and electrons, which are initially created with identical positions. For the gas densities and laser parameters considered here, this approximate way of accounting for the decrease in the field energy due to ionization does not introduce a significant error. 

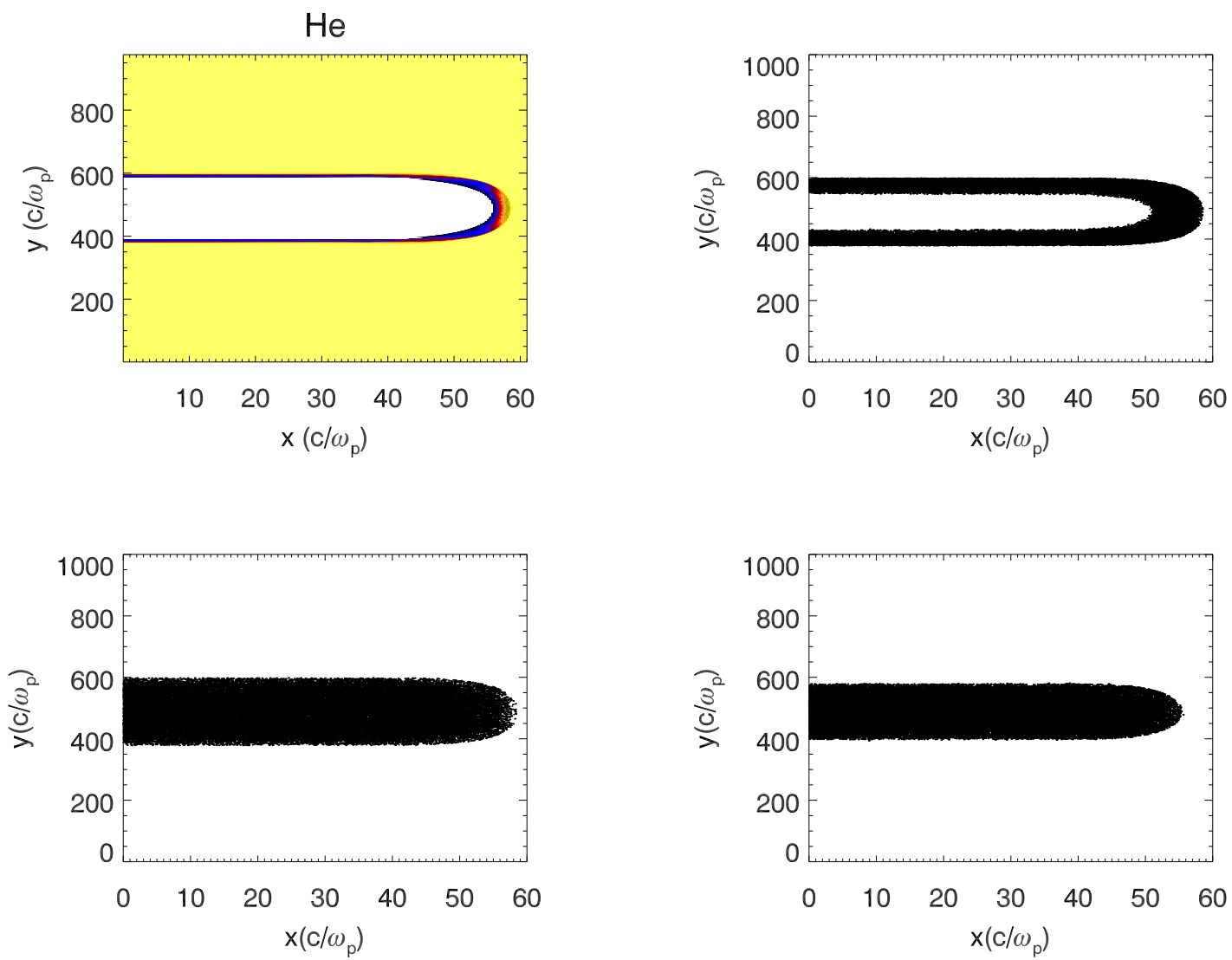

FIGURE 1. Propagation of a laser pulse through a constant He gas density and the associated particle plots showing the effect of the tunneling ionization.

Currently, the implementation has been completed for $\mathrm{H}, \mathrm{He}$, and Li. An example of a constant gas density for $\mathrm{He}$ and the particle plots for electrons, $\mathrm{He}+$ and $\mathrm{He}++$ due to the propagation of a laser pulse simulated with OOPIC is given in Fig. 1. The simulation is in Cartesian coordinates and the pulse moves from the left to right. It has a Gaussian shape in the transverse direction and enough intensity in its core to completely ionize $\mathrm{He}+$ while at the tails it can only ionize the neutral He atoms.

The code design for handling tunneling ionization in OOPIC is based on the objectoriented capabilities provided by $\mathrm{C}++$. We have designed a generic tunneling ionization class that declares the functionality needed from the implementation for any particular gas. The gases for which we wrote concrete implementations $(\mathrm{H}, \mathrm{He}, \mathrm{Li})$ derive from this class. Each derived class also handles the ionization of any ions, created by ionization of its neutral atoms, that can be further ionized. For example, the implementation for $\mathrm{He}$ is responsible for the ionization of both $\mathrm{He}$ and $\mathrm{He}+$. The object-oriented nature of the code allows an efficient and direct way to add ionization capabilities for other gases when the need for them arises. OOPIC allows the simulations of tunneling ionization when more than one neutral gases are present. This is completely controlled from the input file which also provides basic means to specify a functional dependence for a 
neutral gas profile at the beginning of the simulations.

\section{LASER PULSE FREQUENCY UP-SHIFTING AND TUNNELING IONIZATION SIMULATIONS}

In this section we describe the experimental and simulation parameters, the diagnostics we use to detect and measure frequency up-shifting, the results from the simulations and how they compare with the measured data from the l'OASIS experiments $[1,2]$. The l'OASIS Group has proposed and is using the monitoring of ionization blue shifting at relatively low laser power as a method for determining optimized compression of the laser pulses in a chirped-pulse amplification laser system.

\section{Relevant Experimental Parameters}

A He gas jet with a Gaussian profile is used in the experiments. Its width is $3 \mathrm{~mm}$ which is approximately 22 Rayleigh lengths. The maximum gas density is $2 \times 10^{19}$ $\mathrm{cm}^{-3}$ leading to a peak plasma density of $4 \times 10^{19} \mathrm{~cm}^{-3}$. The laser pulse is linearly polarized with a Gaussian profile, both transversely and longitudinally. The experiments considered here used moderate intensity laser pulses, with a total energy of $20.5 \mathrm{~mJ}$ and full width at half maximum (FWHM) pulse lengths between 50 and 200 fs. The laser wavelength $\lambda$ is $800 \mathrm{~nm}$, a frequency of $2.355 \times 10^{15} \mathrm{rad} / \mathrm{s}$, the waist size is $6 \mu \mathrm{m}$ with the Rayleigh length approximately $R_{L} \approx 141 \mu \mathrm{m}$ in vacuum.

\section{Simulation Parameters}

We conducted 2D simulations in $x-y$ slab geometry with $x$ as the longitudinal direction, the direction of propagation of the laser pulse. The polarization of the pulse is along $z$, the ignorable coordinate. The pulse is launched from the left boundary (constant value of $y$ ) of the simulation box and propagates to the right. The description of how laser pulses are launched in OOPIC is given by Bruhwiler et al. [10]. OOPIC has a moving window algorithm that enables propagation of the laser pulse over a distance greater than the longitudinal length of the simulation box. The laser pulse in the simulations has a Gaussian profile along the transverse directions and a half-sine along the direction of propagation. The half-sine along $x$ differs from the shape of the pulse in the experiments but this choice was made to make the simulation box of manageable size for practically executable runs both in terms of available CPU time and memory, including also runs done on multi-processor computers.

The longitudinal and transverse grid sizes are respectively $d x=0.05 \mu \mathrm{m}$ and $d y=$ $0.8 \mu \mathrm{m}$. This leads to a spatial resolution of $16(\lambda / d x)$. The Courant condition must be satisfied by the time step, leading to $d t=1.2 \times 10^{-16} \mathrm{~s}$ and time resolution of $1 /(d t v)=31$. We did runs with 50,100, and 200 fs FWHM pulse lengths. The grid length along $x$ is chosen large enough to provide containment of the laser pulses and not 
to introduce any significant boundary effects. Simulations were done with a number of grid cells $N_{x}=1024,1536,3072$ along $x$ and $N_{y}=1024$ along $y$. The pulse is initially launched in vacuum, approximately one longitudinal grid length $L_{x}$, until it is completely contained in the simulation box. Then it enters the domain of the He gas. The gas profile is a linear ramp increasing in a small number of grid cells along $x$ from zero to a constant gas density equal to the maximum gas density of the experiments. Once the maximum gas density is reached, it is then kept constant for the rest of the simulations. The pulse is evolved for $12 R_{L}$ to achieve a propagation through the same integrated gas density as in the experiments. Propagating the laser pulses half the distance through the peak gas density helped keep memory requirements and CPU times to manageable levels, but this approach leads to some important differences, such as self-modulation of the pulses. In future work, we will model the same gas density profile as in the experiments. The waist location is at $6 R_{L}+L_{x}$.

\section{Blue Shifting and Ionization-induced Pump Depletion for Laser Pulses Ionizing He}

The detection of the blue shifting signatures in the simulations required the development of additional real-time and post-processing diagnostics for OOPIC. In the l'OASIS experiments, the integrated power spectrum of the intensity is measured. The $10 \%$ level decrease of this spectrum (on the shorter wavelength side) from its peak at $800 \mathrm{~nm}$ is used as a measure for the frequency up-shifting. We implemented a similar calculation in the code.

The power spectrum of a field component is calculated, using the fast Fourier transformation, for each lineout of the component with a constant value of $y$ and along $x$, in our case $E_{z}(x, y=$ const $)$. The resulting power spectrum is integrated along a subinterval of the $y$ axis to obtain the integrated power spectrum that can then be compared with the experimental results. The reason to use a subinterval of the simulation grid along $y$ is an attempt to account for the fact that in the experiments only the main part of the pulse is measured by the detectors. The measure of the blue shifting is done integrating over the pulse power spectrum through the middle of the pulse. This domain captures the major part of the laser pulse and neglects the transverse wings, which are expected to be refracted away before the pulse enters the spectrometer.

The surface plot of the $E_{z}$ component of the electric field after the pulse has traveled the length of $12 R_{L}+2 L_{x}$ is shown in Fig. 2, the top left plot. The FWHM pulse length is $100 \mathrm{fs}$. The simulations are designed to launch the pulse first in vacuum, propagate it for one box length $L_{x}$, then propagate it for $12 R_{L}$ in the gas (the same integrated gas density as in the experiments), and finally to switch off the gas and propagate the pulse for another $L_{x}$ distance before a measurement of the blue shifting value is done.

The lineout of $E_{z}$ through the middle of the pulse is shown in the top right plot in Fig. 2. This plot displays more clearly two very interesting effects that have developed due to the tunneling ionization and the interaction with the plasma. First, the ionization happens at the foot of the pulse where the field intensity starts its increase from zero, reaches the interval for which the ionization occurs, and then exceeds it. In the region 

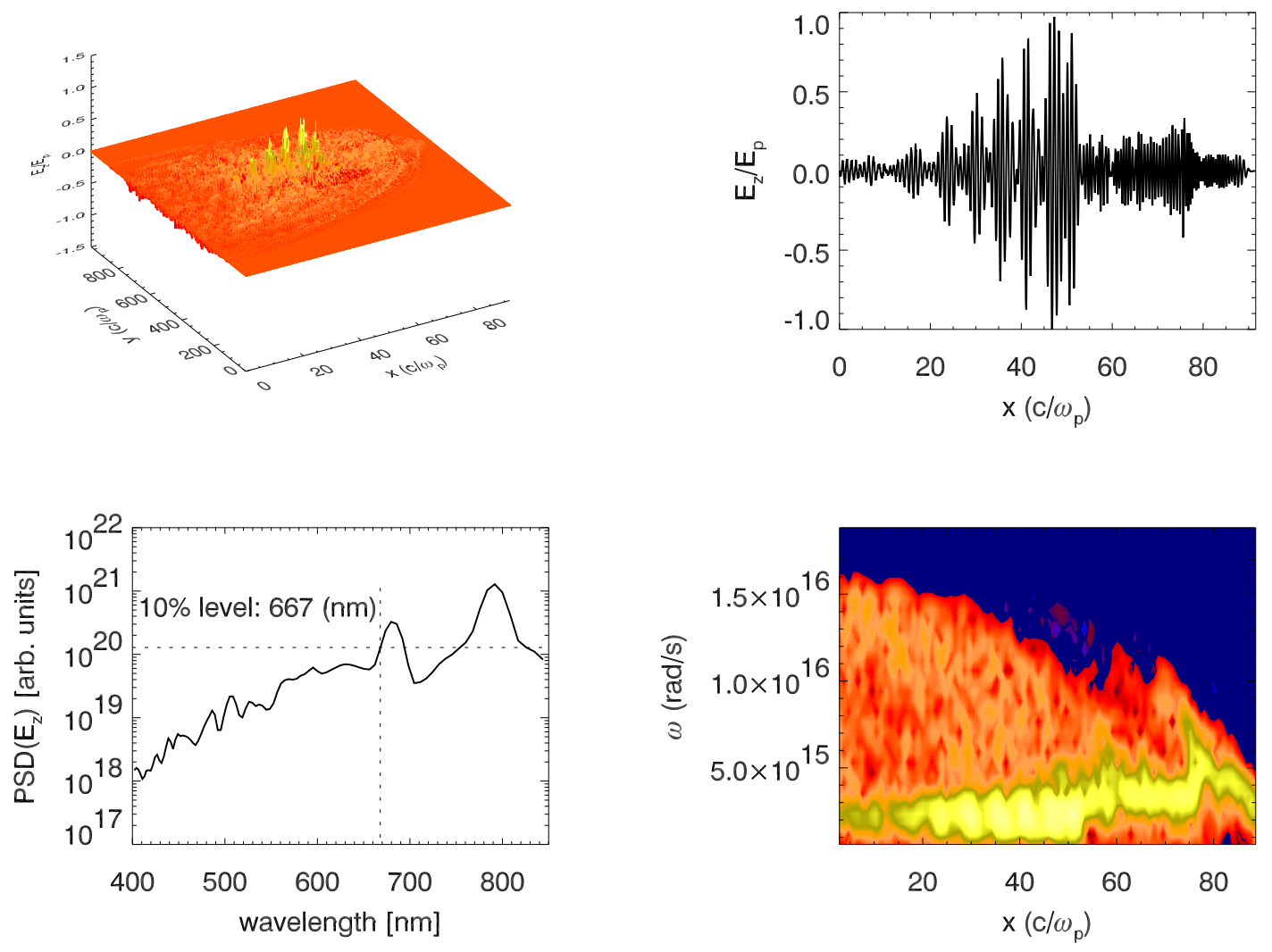

FIGURE 2. Plots, from left to right and top to bottom, of the $E_{z}$ component of the electric field, a lineout through the middle of the pulse, the $E_{z}$ power spectrum, and the $E_{z}$ chirp spectrogram after propagation length of $12 R_{L}+2 L_{x}$.

of the ionization, a considerable ionization-induced pump depletion is observed and the pulse shows a steep rise at the end of this depletion region. The ionization also leads to the blue shifting of the pulse frequency in the pulse domain where the ionization occurs, due to the gradient in the electron density. We have spatially resolved this effect and describe it below. Second, the lineout shows a significant self-modulation of the pulse.

The integrated power spectrum of $E_{z}$ is shown in the bottom left plot of Fig. 2. The $10 \%$ level of decrease from the laser wavelength peak at $800 \mathrm{~nm}$ gives a blue shifting value of $667 \mathrm{~nm}$. This level was determined by the first point on the power spectrum that reaches the $10 \%$ decrease level from the level of the $800 \mathrm{~nm}$ peak moving from the high frequency part of the spectrum (low wavelengths) toward the peak. The well formed peak that appears at approximately $680 \mathrm{~nm}$ is the first harmonic of the laser pulse modulation due to forward Raman scattering.

The qualitative behavior of spatially resolving the region where blue shifting occurs can be deduced from the lineout plot of $E_{z}$ shown in Fig. 2. We have also used the lineout data to obtain a quantitative signature that resolves spatially, along $x$, the blue shifting. We calculate the chirp spectrogram from the data by selecting a window of the data and 
calculating the power spectrum for different windows. This produces a power spectrum lineout that is plotted for the middle point of the interval of $x$ points for each window. We used a window width of 64 points and a shift of 16 points between neighboring windows. The resulting power spectrum lineouts can be plotted together as a surface or contour plot in the frequency-position plane. The contour plot of the chirp spectrogram for the $100 \mathrm{fs}$ run is shown in the bottom right plot of Fig. 2.

It is clear from this plot that the frequency content of the very front of the pulse moves from the highest blue shift values toward the original frequency of the pulse. The lightest band along the bottom of this plot represents the highest power spectrum intensities. This part of the pulse, in the far right region for $x \omega_{p} / c>80$, is propagating effectively at the speed of light in vacuum. It does not have enough intensity to ionize the gas. The spike at $x \omega_{p} / c=75$ is due to an electron density gradient where ionization of $\mathrm{He}$ to $\mathrm{He}+$ occurs. For $55<x \omega_{p} / c<75$, blue-shifted fields see a weaker gradient due to $\mathrm{He}+$ to He++ ionization and at $x \omega_{p} / c=55$ ionization stops since He+ is completely ionized. The main pulse moves with a group velocity less than the speed of light in constant electron density of $4 \times 10^{19} \mathrm{~cm}^{-3}$ for $x \omega_{p} / c<55$ and no blue shifting occurs.

\section{Comparison with Experimental Results}

We compared the results for the blue shifting values from our simulations with the experimental results for runs with FWHM pulse lengths of 50, 100, and 200 fs. We did serial runs at 50 and 100 fs lengths and parallel runs on the Tech-X Corporation Linux cluster for 50 and 200 fs length pulses. The experimental data and the simulation results for the frequency up-shifting values are shown in Fig. 3. These results show good agreement with the experimental measurements. They properly reproduce the functional behavior seen in the experiments that shorter, higher-intensity pulses lead to more blue shifting.

The serial and parallel runs differ due to the different boundary conditions used (not all boundary conditions implemented in serial are also implemented in parallel) the different way random numbers are used in serial and parallel, and the use of particle limits in serial. The serial runs are done with wave-absorbing boundary conditions at the transverse boundaries to minimize reflections. Perfectly conducting boundary conditions had to be used for the parallel runs. We are currently resolving these differences.

\section{Comparison of Tunneling Ionization with Preionized Plasma Simulations}

The simulation of a laser pulse propagating in preionized plasma is of considerable importance for comparing with the tunneling ionization results. The blue shifting is an effect caused by the interaction of the laser field with the co-propagating ionization front. We show in Fig. 4 the lineouts of the $E_{z}$ component of the field through the middle of the pulse for both tunneling ionization and pre-ionized OOPIC runs (the top two plots from left to right). This is a snap shot from the simulations after propagating for $6 R_{L}$ in the 


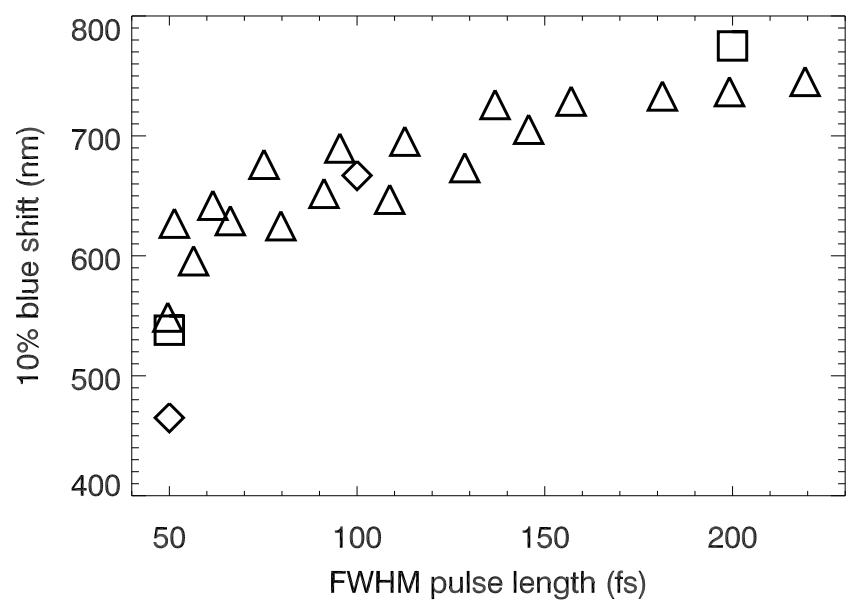

FIGURE 3. Laser frequency up-shifting values from the experiments and the OOPIC simulations. Triangles represent the experimental points, diamonds the simulation results from serial runs and squares the points from parallel runs.
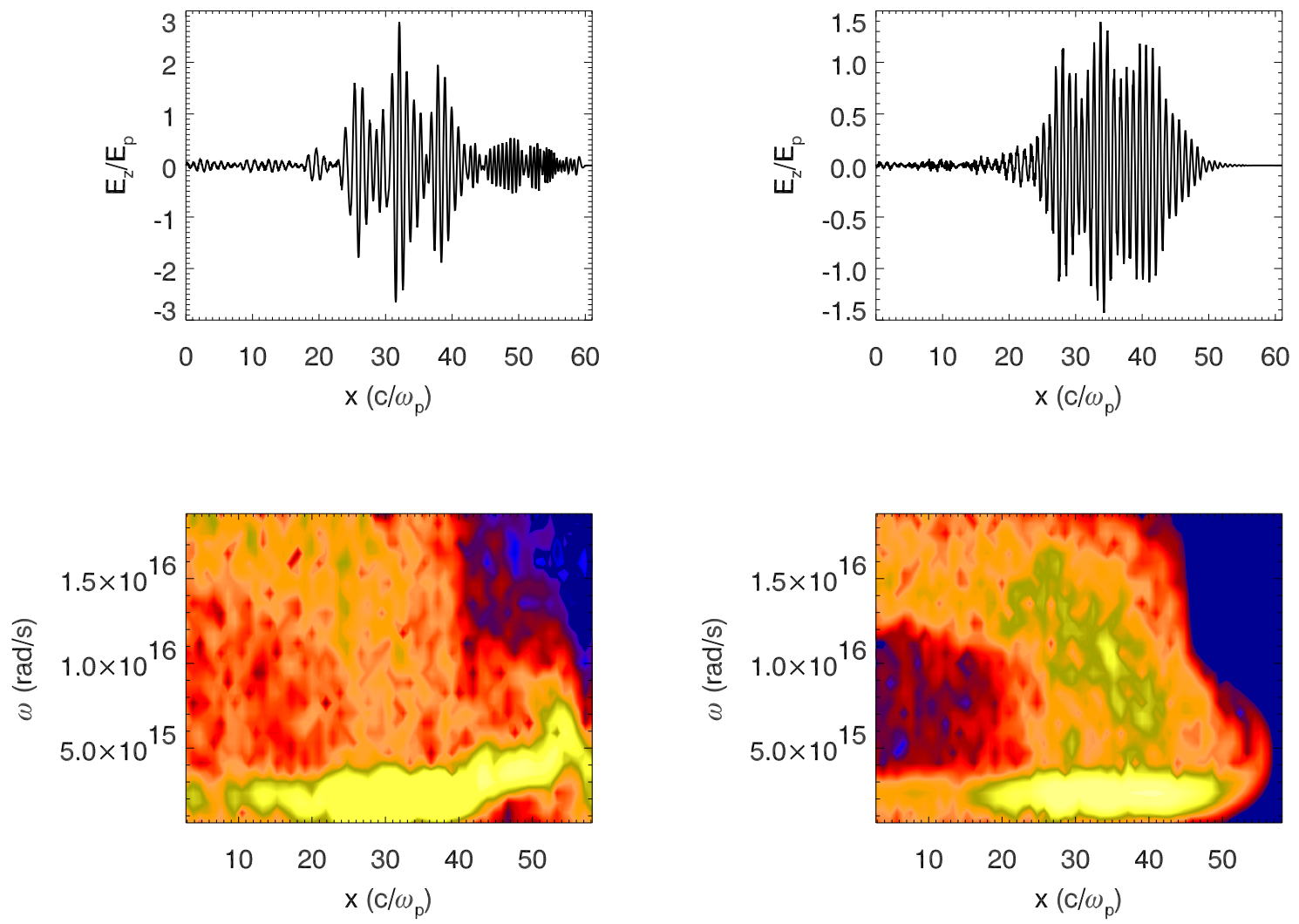

FIGURE 4. Comparison of the tunneling ionization effects on a laser pulse with the corresponding behavior when the pulse propagates in pre-ionized plasma. 
gas and the pre-ionized plasma. Only the lineout of $E_{z}$ in the gas shows the ionizationinduced pump depletion effect and the frequency up-shifting at the front of the pulse. Both plots show self-modulation of the pulse due to the interaction with the plasma. The modulation is much stronger in the tunneling ionization case, consistent with the idea that ionization-induced pump depletion can seed this instability. The two bottom plots in Fig. 4 display the spatially resolved frequency contents of the two $E_{z}$ lineout plots. These chirp spectrograms show blue-shifting of the pulse when propagating in the gas and the absence of this effect in the pre-ionized case.

\section{SUMMARY AND FUTURE WORK}

The frequency up-shifting seen in OOPIC simulations of the LBNL l'OASIS experiments agrees quantitatively with the experimental data. Comparison of OOPIC runs on laser-plasma interactions with and without tunneling ionization show that for preionized plasmas no blue-shifting or ionization-induced pump depletion is seen. Self-modulation of the the laser pulse is seen in both the tunneling ionization and the pre-ionized runs. A continuing priority for rigorous validation against the measurements is the simulation of laser pulses propagating for 22 Rayleigh lengths through the same gas jet density profile as in the l'OASIS experiment. A study to resolve the differences seen between parallel and serial OOPIC runs is currently under way.

\section{ACKNOWLEDGMENTS}

This work was supported by the US Department of Energy under the DE-FG0201ER41178 SciDAC Accelerator Project and the DE-FG03-99ER82903 (Tech-X Corp.), DE-FG03-95ER40926 (U. Colorado), DE-AC03-76SF00098 (LBNL), and also the use of Cray T3E and IBM SP computers at NERSC) grants. The authors acknowledge helpful discussions with J. Verboncoeur, P. Mardahl, and R. Busby regarding OOPIC code development and data analysis.

\section{REFERENCES}

1. Leemans, W. P., Catravas, P., Esarey, E., Geddes, C. G. R., Trines, C. T. R., Schroeder, C. B., Shadwick, B. A., van Tilborg, J., and Faure, J. (in preparation).

2. Leemans, W. et al., "Laser Wakefield Accelerator Experiments at LBNL," in Advanced Accelerator Concepts: Ninth Workshop, edited by P. L. Colestock and S. Kelly, American Institute of Physics, 2001, pp. 136-145.

3. Esarey, E., Joyce, G., and Sprangle, P., Phys. Rev. A, 44, 3908-3911 (1991).

4. Esarey, E. et al., IEEE Trans. Plasma Sci., 24, 252 (1996).

5. Corkum, P. B., IEEE J. Quantum Electron., 21, 216 (1985).

6. Wood, W. M., Focht, G., and Downer, M., Opt. Lett., 13, 984 (1988).

7. Gordon, D. et al., Phys. Rev. E, 64, 046404 (2001).

8. Leemans, W. P. et al., Phys. Rev. A, 46, 1091 (1992).

9. Verboncoeur, J. P., Langdon, A. B., and Gladd, N. T., Comp. Phys. Comm., 87, 199-211 (1995). 
10. Bruhwiler, D. L., Giacone, R. E., Cary, J. R., Verboncoeur, J. P., Mardahl, P., Esarey, E., Leemans, W. P., and Shadwick, B. A., Phys. Rev. ST Accel. Beams, 4, 101302 (2001).

11. QScimpl: A Qt-based Scientific Modeling and Plotting Library (2002), URL http://www . techxhome.com/products/qscimpl/index.html.

12. Cary, J. C., and Nieter, C., "VORPAL: a arbitrary dimensional hybrid code for computation of pulse propagation in laser-based advanced acceleration concepts," in 18th Annual Review of Progress in Applied Computational Electromagnetics ACES 2002 (Monterey, CA), 2002, pp. 549-555.

13. Nieter, C., and Cary, J. C., "VORPAL as a Tool for the Study of Laser Pulse Propagation in LWFA," in Proc. ICCS 2002, Lecture Notes in Computer Science, edited by P. Sloot, C. Tan, J. Dongarra, and A. Hoekstra, Springer-Verlag (Berlin Heidelberg New York), 2002, vol. 2331, p. 334.

14. Vahedi, V., and Surendra, M., Comp. Phys. Comm., 87, 179-198 (1995).

15. Keldysh, L. V., Sov. Phys. JETP, 20, 1307 (1965).

16. Perelomov, A. M., Popov, V. S., and Terent'ev, M. V., Sov. Phys. JETP, 23, 924 (1966).

17. Ammosov, M. V., Delone, N. B., and Krainov, V. P., Sov. Phys. JETP, 64, 1191 (1986).

18. Delone, N. B., and Krainov, V. P., Multiphoton Processes in Atoms, Springer Series on Atoms + Plasma v. 13, Springer-Verlag, Berlin-Heidelberg, 2000.

19. Landau, L. D., and Lifshitz, E. M., Quantum Mechanics: Non-Relativistic Theory, Pergamon, Oxford, 1977. 